\title{
Serum urate and lung cancer: a cohort study and Mendelian randomization using UK Biobank
}

\author{
Laura J. Horsfall ${ }^{* *}$, lan P. Hall ${ }^{2,3}$ and Irwin Nazareth ${ }^{1}$
}

\begin{abstract}
Background: Serum urate is the most abundant small molecule with antioxidant properties found in blood and the epithelial lining fluid of the respiratory system. Moderately raised serum urate is associated with lower rates of lung cancer and COPD in smokers but whether these relationships reflect antioxidant properties or residual confounding is unknown.

Methods: We investigated the observational and potentially causal associations of serum urate with lung cancer incidence and FEV using one-sample Mendelian randomization (MR) and the UK Biobank resource. Incident lung cancer events were identified from national cancer registries as $\mathrm{FEV}_{1}$ was measured at baseline. Observational and genetically instrumented incidence rate ratios (IRRs) and risk differences per 10,000 person-years (PYs) by smoking status were estimated.
\end{abstract}

Results: The analysis included 359,192 participants and 1,924 lung cancer events. The associations between measured urate levels and lung cancer were broadly U-shaped but varied by sex at birth with the strongest associations in current smoking men. After adjustment for confounding variables, current smoking men with low serum urate (100 $\mathrm{mmol} / \mathrm{L})$ had the highest predicted lung cancer incidence at 125/10,000 PY (95\% Cl 56-170/10,000 PY) compared with 45/10,000 PY (95\%Cl 38-47/10,000 PY) for those with the median level (300 $\mu \mathrm{mol} / \mathrm{L})$. Raised measured urate was associated with a lower baseline $\mathrm{FEV}_{1}$. The MR results did not support a causal relationship between serum urate and lung cancer or $\mathrm{FEV}_{1}$.

Conclusions: We found no evidence that serum urate is a modifiable risk factor for respiratory health or lung cancer. Keywords: Mendelian randomization, Urate, Lung function, Causal relationship, Lung cancer, Smoking phenotypes

\section{Background}

Purine compounds, including adenine and guanine, are essential for many cell processes. Although it is possible to synthesise purines, animals derive large quantities from food, including certain meats and seafood. Excess dietary purines are transported in blood to the liver where they are converted (oxidised) to urea and allantoin

\footnotetext{
*Correspondence: laura.horsfall@ucl.ac.uk

${ }^{1}$ Research Department of Primary Care and Population Health, University College London, Royal Free Hospital Campus, London NW3 2PF, UK

Full list of author information is available at the end of the article
}

by the enzyme uricase. However, uricase is absent in humans due to loss-of-function mutations in the uricase gene [1], which means urate levels in human blood are around fifty times higher than non-primate mammals [2]. For some people, a purine-rich diet can lead to the condition gout, where urate crystals accumulate in joints causing pain and inflammation. However, the reduction and loss of uricase activity during higher primate evolution together with some unusual features of urate metabolism, has led to speculation that raised levels may also benefit humans physiologically [3-5].

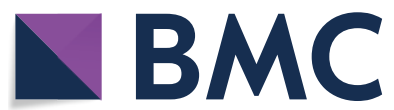

(c) The Author(s) 2021. Open Access This article is licensed under a Creative Commons Attribution 4.0 International License, which permits use, sharing, adaptation, distribution and reproduction in any medium or format, as long as you give appropriate credit to the original author(s) and the source, provide a link to the Creative Commons licence, and indicate if changes were made. The images or other third party material in this article are included in the article's Creative Commons licence, unless indicated otherwise in a credit line to the material. If material is not included in the article's Creative Commons licence and your intended use is not permitted by statutory regulation or exceeds the permitted use, you will need to obtain permission directly from the copyright holder. To view a copy of this licence, visit http://creativecommons.org/licenses/by/4.0/. The Creative Commons Public Domain Dedication waiver (http://creativeco mmons.org/publicdomain/zero/1.0/) applies to the data made available in this article, unless otherwise stated in a credit line to the data. 
Serum urate has powerful antioxidant properties in vitro and, with an average concentration of $300 \mu \mathrm{mol} / \mathrm{L}$, it is the most abundant molecule with antioxidant properties present in human blood [6, 7]. Estimates suggest as much as $50 \%$ of human blood antioxidant capacity is accounted for by the action of serum urate [8]. This has led to theories that the low cancer rates and longevity in hominids relative to other mammals are partly due to the reduction and loss of uricase activity [9].

As well as being found at high concentrations in blood, urate is found at high concentrations in human respiratory tissues and the epithelial lining fluid of the respiratory tract and could provide an important first-line defence against environmental oxidants from smoke and pollution [10, 11]. Our group's previous large-scale cohort study of people living in the UK found that cigarette smokers with moderately high serum urate had substantially lower rates of COPD and lung cancer [12]. However, the cohort only included people with a urate measure in their primary health care records, which is a highly selective sample and replication in an independent cohort is warranted. Furthermore, the association may reflect residual confounding or reverse causation rather than antioxidant properties of urate.

\section{Methods}

\section{Aims}

The first aim of this study was to see whether we could replicate the association between measured serum urate and respiratory outcomes. The second aim was to examine whether there was any evidence supporting a causal association with genetically predicted serum urate and respiratory cancer(Mendelian randomization). We also examined the associations between urate and respiratory function as a phenotype that might also be influenced by endogenous antioxidant activity.

\section{Data source}

We used The UK Biobank Resource, a prospective cohort study of over 500,000 participants aged 40-69 years, recruited between 2006 and 2010 from around the UK [13]. Further information on UK Biobank such as the processing of biological samples including DNA is available at the following: https://www.ukbiobank.ac.uk/. The quality control and imputation of SNPs, indels and structural variants are reported elsewhere [14].

\section{Study design}

The methods we report are similar to our earlier study on serum bilirubin using UK Biobank [15]. In brief, we analysed the longitudinal relationship between serum urate levels and lung cancer and the cross-sectional relationship between serum urate and forced expiratory volume in $1 \mathrm{~s}\left(\mathrm{FEV}_{1}\right)$. We estimated the causal relationships between urate levels and these outcomes by applying Mendelian randomization (MR) to individual-level data. The protocol was approved by UK Biobank in July 2018 (ID:5167) and we checked the adequacy of sample size using online tools (http://cnsgenomics.com/shiny/ $\mathrm{mRnd} /$ ).

\section{Inclusion/exclusion criteria}

We excluded people who no longer wished to participate in UK Biobank up to August 2020 and applied several genetic exclusions including outliers for genotype missingness or excess heterozygosity, sex aneuploidy and sex discordance $(n=2200)$. We used a published algorithm to retain unrelated participants [16] $(\mathrm{n}=39,642)$ and finally restricted the sample to "white British" participants based on self-reported ethnic identity and principal components available in the dataset $(n=88,341)$ [14]. We set the cohort start date at the date when the participant attended the research centre and the exit date was the earliest date of lung cancer diagnosis, loss to follow-up, death or end of the follow-up period. At the time of analysis, the most recent date for complete follow-up for incident cancers was March 2016 for England and Wales and October 2015 for Scotland. Prevalent lung cancer cases were excluded $(n=512)$.

\section{Exposures}

Almost all participants provided blood samples at the initial assessment centre visit. Serum urate was assayed in theses samples by Uricase PAP (Beckman Coulter AU5800). We selected 31 SNPs for estimating genetically predicted urate levels based on the results of a large-scale Genome Wide Association Analysis (GWAS) of European people [17]. The two lead GWAS SNPs (rs12498742 and rs2231142) are located in renal and gut urate transporters [18] and we analysed these separately as well as in combination with the 28 weaker variants.

\section{Outcomes}

The primary outcome was a new lung cancer diagnosis recorded after study recruitment. Cancer diagnoses in UK Biobank are provided by the NHS Central Register for participants living in Scotland and the Health \& Social Care Information Centre for participants living in England and Wales. Diagnoses are coded using the International Classification of Disease (ICD) version 9 and 10 and we selected malignant neoplasms of the trachea and bronchus (ICD10: C33-C34) as the cancers where smoking has the strongest pathophysiological role and highest attributable risk [19]. In addition to the national cancer registries, we used self-reported cancer diagnosis to identify prevalent cancers. 
Table 1 Baseline characteristics of UK Biobank participants by sex-specific quintiles of serum urate

\begin{tabular}{|c|c|c|c|c|c|c|}
\hline \multirow[b]{2}{*}{ Men } & \multirow[t]{2}{*}{ Total } & \multicolumn{5}{|c|}{ Quintile of serum urate $(\mu \mathrm{mol} / \mathrm{L})$} \\
\hline & & 89-294 & $295-332$ & $333-367$ & $368-411$ & $411-600$ \\
\hline \multirow[t]{2}{*}{ Women } & & $89-215$ & $216-248$ & $249-279$ & $280-321$ & $322-600$ \\
\hline & $N=359,192$ & $N=71,716$ & $N=71,889$ & $N=71,745$ & $N=71,927$ & $N=71,915$ \\
\hline Sex & $166,618(46.4 \%)$ & $33,296(46.4 \%)$ & $33,320(46.3 \%)$ & $33,278(46.4 \%)$ & $33,380(46.4 \%)$ & $33,344(46.4 \%)$ \\
\hline Age at recruitment (IQR) & $58.9(51.3-64.0)$ & $57.3(49.4-63.1)$ & $58.1(50.3-63.5)$ & $58.7(51.3-63.8)$ & $59.5(52.3-64.2)$ & $60.5(53.7-64.9)$ \\
\hline Weight (kg) & $78.3(15.8)$ & $72.3(14.0)$ & $75.1(14.5)$ & $77.7(14.9)$ & $80.6(15.5)$ & $85.8(16.5)$ \\
\hline Height (cm) & $168.8(9.2)$ & $169.0(9.0)$ & $169.0(9.1)$ & $168.9(9.2)$ & $168.8(9.3)$ & $168.4(9.4)$ \\
\hline BMI & $27.4(4.7)$ & $25.2(3.9)$ & $26.2(4.0)$ & $27.1(4.2)$ & $28.2(4.6)$ & $30.2(5.2)$ \\
\hline \multicolumn{7}{|l|}{ Smoking status } \\
\hline Never & $195,303(54.4 \%)$ & $41,107(57.3 \%)$ & $40,526(56.4 \%)$ & $39,585(55.2 \%)$ & $38,386(53.4 \%)$ & $35,699(49.6 \%)$ \\
\hline Former & $126,440(35.2 \%)$ & $21,763(30.3 \%)$ & $23,462(32.6 \%)$ & $24,988(34.8 \%)$ & $26,581(37.0 \%)$ & $29,646(41.2 \%)$ \\
\hline Current & $36,227(10.1 \%)$ & $8622(12.0 \%)$ & $7654(10.6 \%)$ & $6951(9.7 \%)$ & $6726(9.4 \%)$ & $6274(8.7 \%)$ \\
\hline Missing & $1222(0.3 \%)$ & $224(0.3 \%)$ & $247(0.3 \%)$ & $221(0.3 \%)$ & $234(0.3 \%)$ & $296(0.4 \%)$ \\
\hline Pack years of smoking $(\mathrm{IQR})^{*}$ & $19.5(10.0-32.5)$ & $18.8(9.3-31.5)$ & $18.0(9.0-30.6)$ & $18.8(9.5-31.5)$ & $19.5(10.1-32.5)$ & $22.0(12.0-35.5)$ \\
\hline History of lung cancer & $528(0.15 \%)$ & $101(0.14 \%)$ & $82(0.11 \%)$ & $98(0.14 \%)$ & $91(0.13 \%)$ & $156(0.22 \%)$ \\
\hline Family history of lung cancer & $46,291(12.9 \%)$ & $8547(11.9 \%)$ & $8738(12.2 \%)$ & $9218(12.8 \%)$ & $9596(13.3 \%)$ & $10,192(14.2 \%)$ \\
\hline History of COPD/emphysema & $8167(2.3 \%)$ & $1486(2.1 \%)$ & $1458(2.0 \%)$ & $1515(2.1 \%)$ & $1646(2.3 \%)$ & $2062(2.9 \%)$ \\
\hline
\end{tabular}

All continuous variables are mean values with \pm 1 standard or medians for skewed data if interquartile ranges (IQRs) are specified

*Previously calculated for 109,312 participants reporting to regularly smoke at least one cigarette/day and who also reported smoking duration

We also examined the relationship with $\mathrm{FEV}_{1}$. Spirometry was performed during the baseline assessment using a Vitalograph Pneumotrac 6800. The participant was asked to record two to three blows (lasting for at least $6 \mathrm{~s})$ within approximately six minutes. We used the maximum value of $\mathrm{FEV}_{1}$ meeting the assessor's acceptability criteria. Further details on spirometry are reported elsewhere [20].

\section{Other variables}

We included important predictors of lung cancer in analyses including age, calendar year, genetic sex, population sub-structure (first 40 principal components) recruitment centre, height, weight and self-reported smoking status [21, 22]. Weight is strongly associated with urate levels and there is evidence that weight is causally associated with lung cancer [23]. Further, the lead GWAS SNP (rs12498742) is located in a gene that has a role in glucose homeostasis $(S L C 2 A 9)$ that could potentially influence weight. Therefore, we examined models with and without this variable. In a subset of people with a history of regular smoking, we further adjusted for waist circumference, exposure to smoke at home, Townsend social deprivation index, antioxidant supplements, alcohol intake and nitrogen dioxide air pollution.

As a supplementary analysis, we also examined whether participants genetically predisposed to raised urate were less likely to have a positive family history of lung cancer and a lower baseline prevalence of chronic obstructive pulmonary disease (COPD).

\section{Interactions}

We fitted models separately for men and women given the different levels average urate levels as well as evidence of differential genetic effects of SNPs on urate levels [24]. We previously reported strong interactions between urate and smoking status with no clear association in non-smokers but strong negative associations in current smokers [12]. We therefore estimated associations by self-reported smoking status (never, former and current) and smoking intensity (1-19 cigarettes per day or 20 or more cigarettes per day) by including multiplicative interaction terms in the models for each sex. Packyears of smoking was available for a subset of participants and we described continuous-by-continuous interactions with urate.

\section{Statistical analyses}

Serum urate levels were divided into sex-specific quintile categories to describe the univariable associations with other covariates. We identified and excluded outlier values for continuous variables using multivariate approach (blocked adaptive computationally efficient outlier nominators algorithm) including age and sex with a $15 \%$ threshold of the chi-squared distribution [25]. To estimate the observational incidence rate ratios (IRRs) per $100 \mu \mathrm{mol} / \mathrm{L}$ increase in serum urate, we used 
Table 2 Mean baseline FEV1 and lung cancer incidence by sex-stratified quintiles categories of serum urate

\begin{tabular}{|c|c|c|c|c|c|c|}
\hline Smoking status & $\begin{array}{l}\text { Sex stratified } \\
\text { quintile of urate* }\end{array}$ & Number & Mean FEV 1 (SD) & $\begin{array}{l}\text { Lung cancer } \\
\text { events }\end{array}$ & $\begin{array}{l}\text { Person years } \\
(\times 10,000 \text { PYs })\end{array}$ & $\begin{array}{l}\text { Lung cancer incidence } \\
\text { rate per } 10,000 \mathrm{PYs} \\
(95 \% \mathrm{Cl})\end{array}$ \\
\hline \multirow[t]{5}{*}{ Overall } & 1 & 71,720 & $2.93(0.76)$ & 398 & 50.4 & $7.9(7.2-8.7)$ \\
\hline & 2 & 71,895 & $2.90(0.77)$ & 366 & 50.4 & $7.3(6.6-8.0)$ \\
\hline & 3 & 71,753 & $2.87(0.78)$ & 325 & 50.6 & $6.4(5.8-7.2)$ \\
\hline & 4 & 71,932 & $2.83(0.78)$ & 353 & 50.6 & $7.0(6.3-7.7)$ \\
\hline & 5 & 71,952 & $2.73(0.79)$ & 482 & 50.6 & $9.5(8.7-10.4)$ \\
\hline \multirow[t]{5}{*}{ Never } & 1 & 41,109 & $2.96(0.75)$ & 48 & 29.1 & $1.7(1.2-2.2)$ \\
\hline & 2 & 40,528 & $2.94(0.77)$ & 52 & 28.6 & $1.8(1.4-2.4)$ \\
\hline & 3 & 39,586 & $2.90(0.78)$ & 51 & 28.0 & $1.8(1.4-2.4)$ \\
\hline & 4 & 38,387 & $2.85(0.78)$ & 56 & 27.2 & $2.1(1.6-2.7)$ \\
\hline & 5 & 35,720 & $2.76(0.79)$ & 56 & 25.2 & $2.2(1.7-2.9)$ \\
\hline \multirow[t]{5}{*}{ Former } & 1 & 21,764 & $2.92(0.75)$ & 146 & 15.2 & $9.6(8.2-11.3)$ \\
\hline & 2 & 23,463 & $2.89(0.75)$ & 139 & 16.4 & $8.5(7.2-10.0)$ \\
\hline & 3 & 24,993 & $2.87(0.76)$ & 142 & 17.5 & 8.1 (6.9-9.5) \\
\hline & 4 & 26,584 & $2.82(0.77)$ & 178 & 18.6 & $9.6(8.3-11.1)$ \\
\hline & 5 & 29,658 & $2.71(0.78)$ & 288 & 20.7 & 13.9 (12.4-15.6) \\
\hline \multirow[t]{5}{*}{ Current } & 1 & 8623 & $2.82(0.82)$ & 204 & 6.0 & $34.0(29.6-39.0)$ \\
\hline & 2 & 7656 & $2.79(0.82)$ & 174 & 5.3 & $32.8(28.3-38.0)$ \\
\hline & 3 & 6953 & $2.77(0.83)$ & 130 & 4.9 & $26.7(22.5-31.7)$ \\
\hline & 4 & 6727 & $2.76(0.84)$ & 118 & 4.7 & $25.2(21.0-30.2)$ \\
\hline & 5 & 6278 & $2.66(0.84)$ & 133 & 4.4 & $30.3(25.6-35.9)$ \\
\hline
\end{tabular}

*See Table 1 for $\mu \mathrm{mol} / \mathrm{L}$ values

multivariable Poisson regression with robust standard errors and age as the time scale. We explored non-linear relationships by applying restricted cubic spline-interpolation using Harrell's default percentiles and selecting the transformation that minimised the Akaike and Bayesian information criteria (AIC/BIC). To easily visualise nonlinear transformations and interactions, we calculated the margins of response as adjusted incidence rates at different levels of urate while holding all other variables at their observed values. We applied a user-written programme for data visualisation [26] and standard errors for marginal effects were calculated using the delta method. We checked for proportionality of associations with the time scale by testing interaction terms. Continuous variables were parameterised as linear and Wald tests were used for calculating $\mathrm{p}$-values for categorical variables and spline transformations. To investigate any reverse causation, we compared the associations after setting the cohort entry date to one and two years after the urate test date.

We estimated the IRRs for lung cancer per $100 \mu \mathrm{mol} / \mathrm{L}$ increase genetically predicted urate using one-sample MR and the two-stage predictor substitution (2SPS) method [21]. Odds ratios for the supplementary outcomes COPD and family history of lung cancer were also estimated using this method. We used a similar approach, the two stage least squares method (2SLS), to estimate the causal cross-sectional relationship per $100 \mu \mathrm{mol} / \mathrm{L}$ increase genetically predicted urate and $\mathrm{FEV}_{1}[21] . \mathrm{FEV}_{1}$ was missing not at random for approximately $25 \%$ of participants and we used inverse probability weighting in an attempt to reduce the impact of any selection bias. After applying the ERS/ATS criteria for $\mathrm{FEV}_{1}$ reproducibility, $\mathrm{FEV}_{1}$ was missing for $50 \%$ of smokers and we decided against this analysis.

Relatives were excluded using an algorithm in $\mathrm{R}$ (v.3.5.1) [16] and all other analyses were done using Stata v.16.1 (Stata Corporation, College Station, Texas).

\section{Results}

Serum urate levels were available for 359,192 participants (Table 1). There were 1924 incident cases of lung cancer diagnosed after recruitment, 15,335 deaths from any cause and 766 participants were lost to follow-up for reasons including emigration. Median follow-up time was 7-years (interquartile range 6-8 years). Men and women 

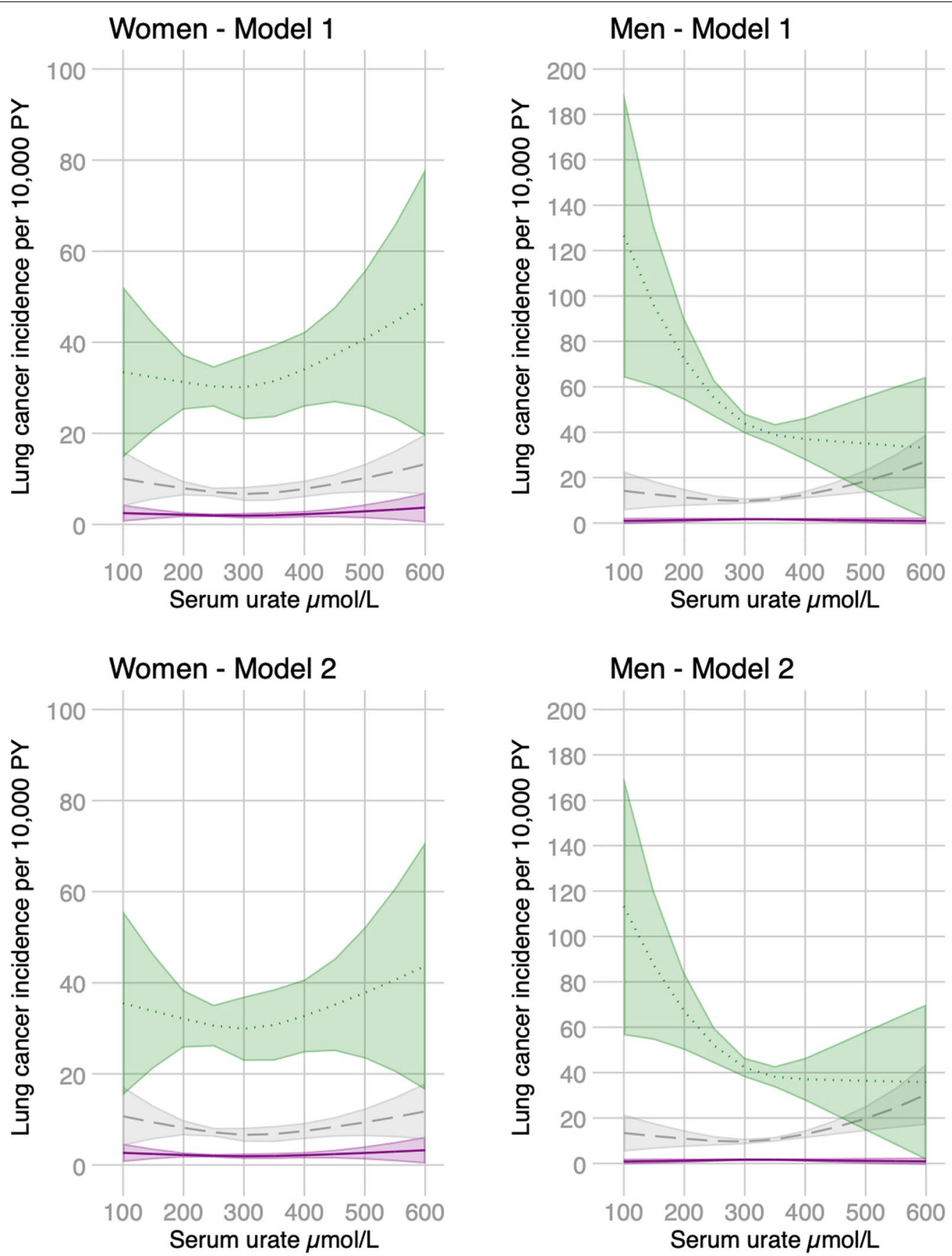

\section{Smoking status}

Fig. 1 Observational associations between serum urate and lung cancer incidence by sex adjusted for age, calendar year, ethnicity (first 40 principal components), height and recruitment centre (Model 1) and additionally for weight (Model 2). Non-linear relationships were captured using restricted cubic spline transformation with three knots placed at the 10th, 50th and 90th percentiles of urate levels 


\section{(See figure on next page.)}

Fig. 2 Observational associations of serum urate with $\mathrm{FEV}_{1}$ and lung cancer incidence in regular smokers by sex adjusted for age, calendar year, ethnicity (first 40 principal components), packyears of smoking, height and recruitment centre (Model 1), additionally for weight (Model 2) and waist circumference, alcohol consumption, exposure to smoke at home, social deprivation, air pollution levels (nitrogen dioxide), and intake of antioxidant supplements (Model 3). Non-linear relationships were captured using restricted cubic spline transformation with three knots placed at the 10th, 50th and 90th percentiles of urate level

with high urate levels were heavier, shorter, more likely to live in socially deprived areas (Table 1). Those in the highest quintile were also more likely to report a clinical diagnosis of lung cancer/COPD and emphysema prior to recruitment (Table 1). However, there were fewer current smokers in the highest quintile. Unadjusted lung function tended to decline as urate increased across smoking categories and the relationship with lung cancer was U-shaped (Table 2).

Observational associations with urate differed by sex and smoking status. There was a weak U-shaped association between observed urate and the incidence of lung cancer in women without strong evidence of multiplicative interactions (Fig. 1). In contrast, we found strong L-shaped relationships between observed urate levels and lung cancer incidence in current smoking men but weaker associations in other smoking categories (Fig. 1). Reverse causation does not fully explain these associations, which remained similar after changing the date of cohort entry to one and two years after the urate measurement (Additional file 1: Fig. S1). We found L-shaped associations with lung cancer for men and women who smoked regularly (at least 1 cigarette per day), and these were slightly attenuated after adjusting for several other variables (Fig. 2). We found continuous by continuous interactions with packyears of smoking and lung cancer where the highest predicted incidence was for men and women with the lowest urate and highest number of pack-years (Fig. 3). Adjusted $\mathrm{FEV}_{1}$ declined across most smoking strata as observed levels of urate increased (Additional file 1: Fig. S2). Although for male current smokers, $\mathrm{FEV}_{1}$ increased up to around $300 \mu \mathrm{mol} / \mathrm{L}$ of urate followed by general decline (Additional file 1: Fig. S2). FEV declined as urate increased in current or former regular smokers and remained unchanged after adjustment (Fig. 3). There was evidence of similar interactions between $\mathrm{FEV}_{1}$ and pack-years with the lowest predicted $\mathrm{FEV}_{1}$ for men and women with the lowest urate and highest number of pack-years (Additional file 1: Fig. S3).

We confirmed that the selected SNPs were associated with urate levels explaining 5.3\% (F statistic $=528$ ) of the variability (Additional file 1: Fig. S4). The onesample MR-analysis 376,922 participants had complete data (directly genotyped or imputed) for the two main
SNPs and 305,614 had complete data for all 31 SNPs. There was no clear pattern in the per allele effects on $\mathrm{FEV}_{1}$ and lung cancer (Additional file 1: Fig. S4). The results of the MR with all 31 SNPs (Table 3) or with the two lead GWAS SNPs (Additional file 1: Table S1) did not support a causal association between urate and $\mathrm{FEV}_{1}$ or lung cancer. Separate analyses by sex and including probability weights for the analysis of $\mathrm{FEV}_{1}$ did not alter our overall conclusions. There was no evidence of an association between genetically predicted urate and a family history of lung cancer or prevalent COPD/emphysema (Additional file 1: Table S2).

\section{Discussion}

\section{Summary}

As far as we know, this is the largest study to examine observational and potentially causal interactions between serum urate, cigarette smoking and lung cancer. Although the incidence of lung cancer was higher at the lowest levels of urate for men and women with a history of smoking and particularly for those with the highest number of pack-years, we found no substantial evidence to support causality. The observational associations, in our view, reflect residual confounding by factors associated with weight or diet. However, as a low cost and simple assay, the finding that associations remained after adjusting for several variables used in lung cancer risk prediction, suggests further work is needed to establish the value of urate in improving risk stratification. Low-dose computed tomography (CT) screening programmes are being adopted in the United States and piloted in the United Kingdom [27]. Even small improvements in risk prediction could have a meaningful impact due to the high mortality burden of lung cancer together with the financial and psychological cost of false-positives of CT-screening.

\section{Comparison with other studies}

We found that higher levels of urate were independently associated with lower levels of $\mathrm{FEV}_{1}$, which is consistent with earlier findings for lung function in healthy people and for exacerbations and mortality associated with a COPD diagnosis [28-30]. In the absence of any strong indication of causality, the higher levels of urate in people with worse $\mathrm{FEV}_{1}$ could reflect reverse causation due to the cross-sectional design. For 


\section{History of regularly smoking cigarettes}

FEV $_{1}$

Model 1

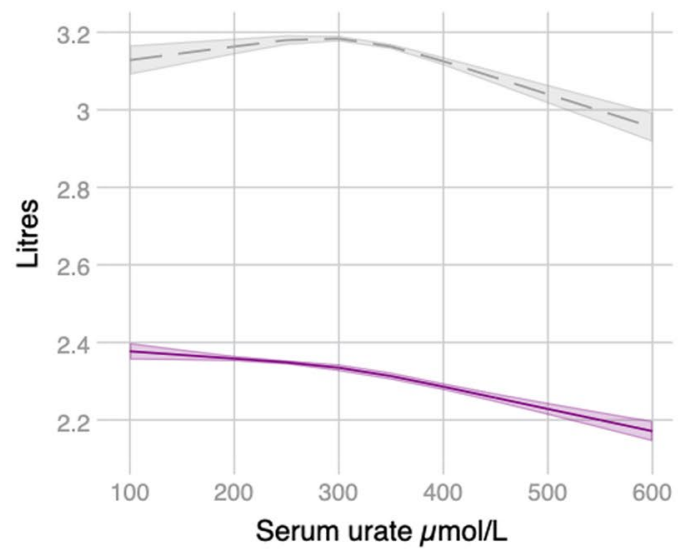

Model 2

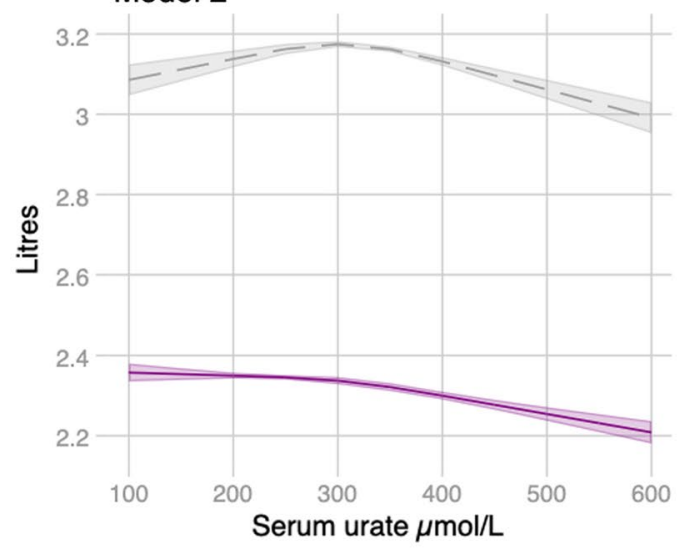

Model 3

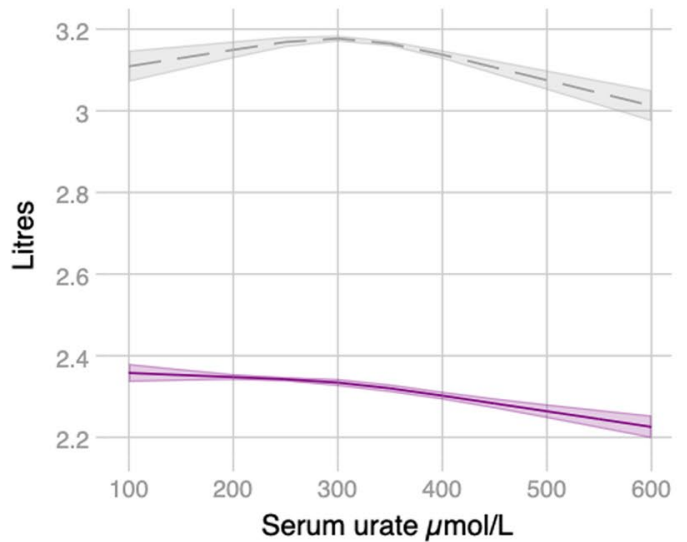

- Women

- - Men
Lung cancer

Model 1

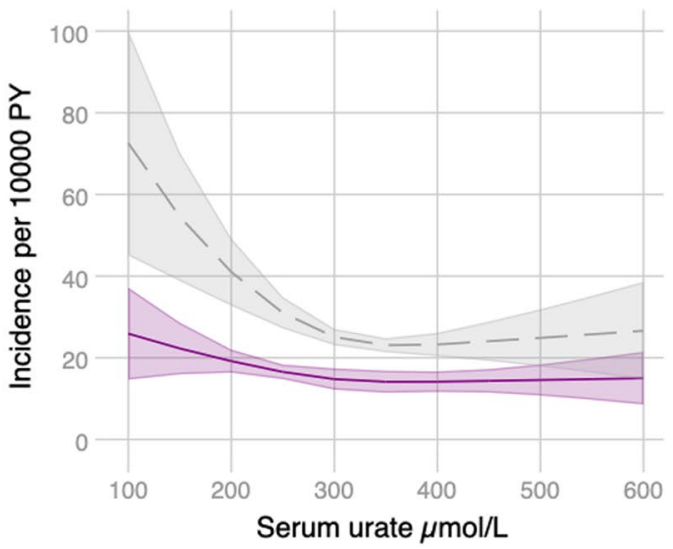

Model 2

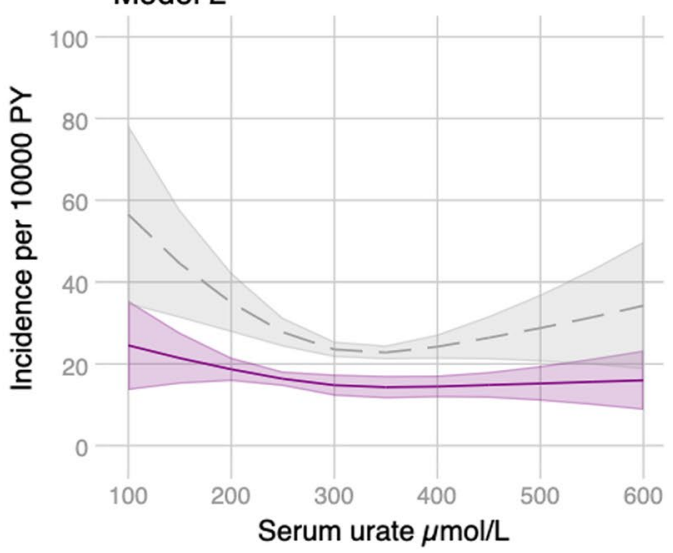

Model 3

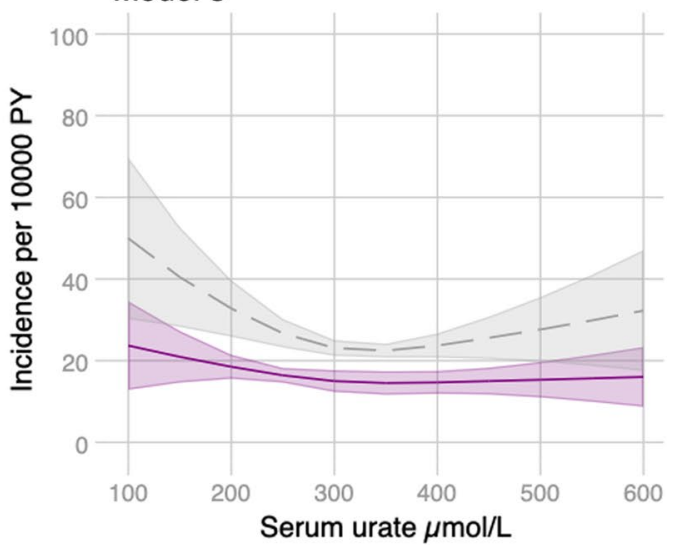

95\% Confidence interval

95\% Confidence interval 

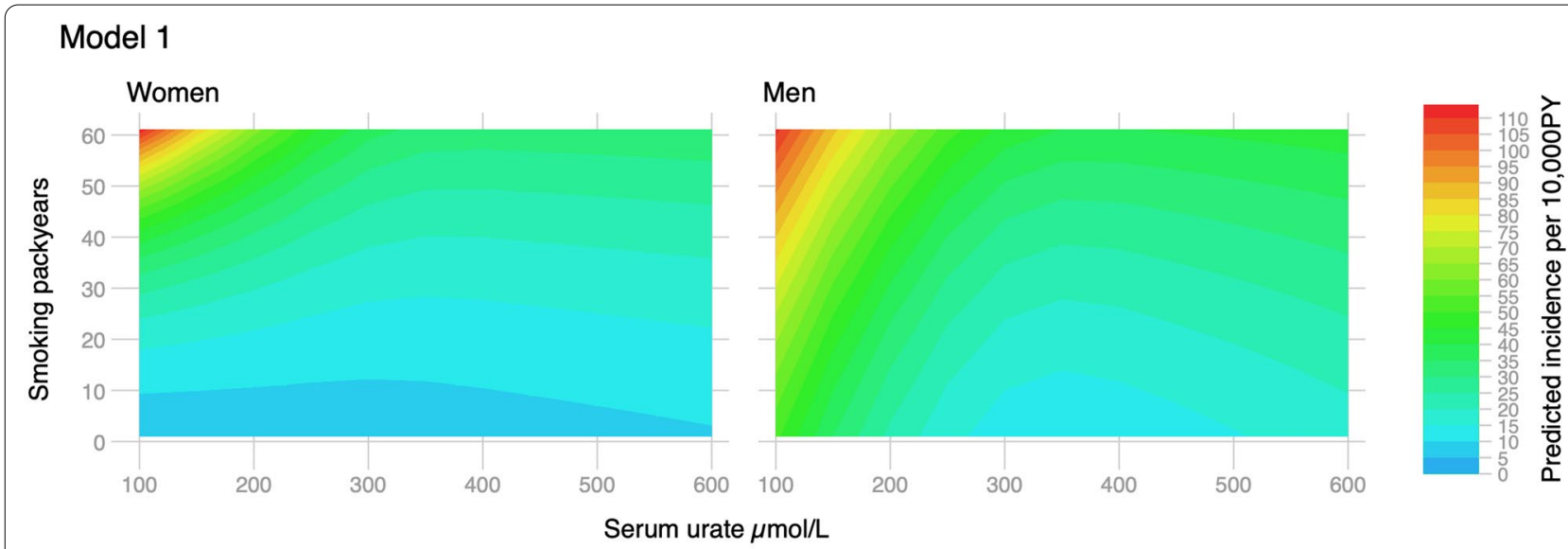

\section{Model 2}
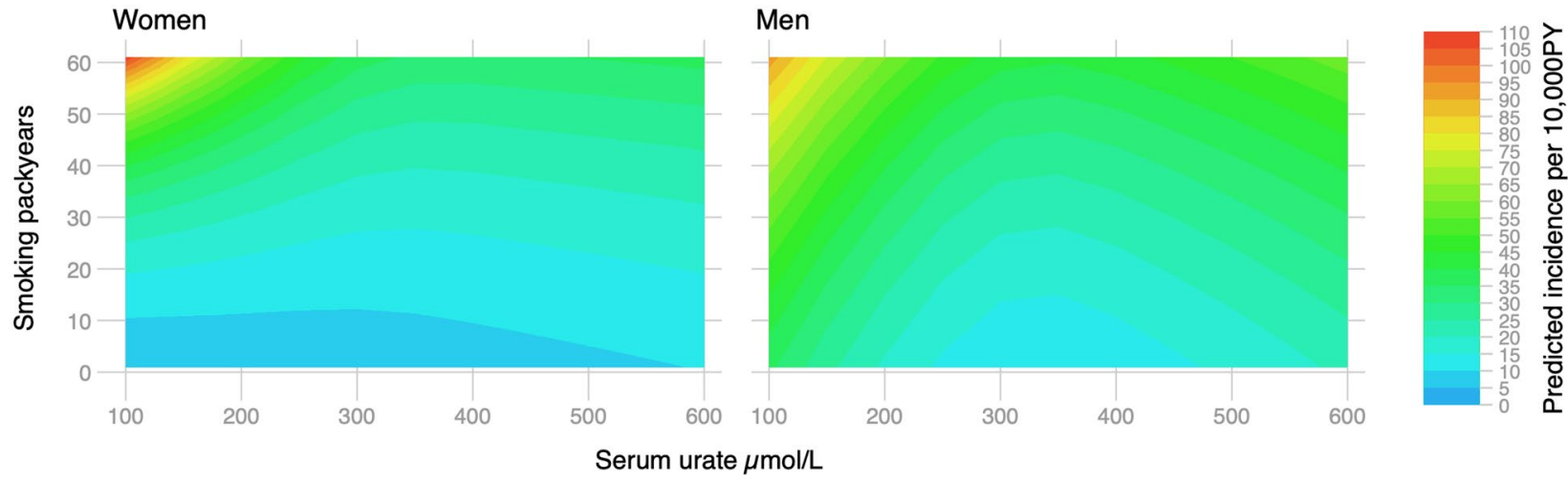

Fig. 3 Observational associations between serum urate and lung cancer incidence with interactions with smoking packyears in regular smokers by sex adjusted for age, calendar year, ethnicity (first 40 principal components), height, recruitment centre (Model 1) and additionally for weight (Model 2). Non-linear relationships were captured using restricted cubic spline transformation with three knots placed at the 10th, 50th and 90 th percentiles of urate level

Table 3 Associations between genetically predicted urate, baseline FEV1 and lung cancer incidence

\begin{tabular}{llllll}
\hline & $\begin{array}{l}\text { Coefficient per } \mathbf{1 0 0} \boldsymbol{\mu m o l} \\
\text { serum urate* } \\
\text { FEV } \mathbf{1}(\mathbf{m l})\end{array}$ & $\mathbf{p}$ value & $\begin{array}{l}\text { Coefficient per } \mathbf{1 0 0} \boldsymbol{\mu m o l} \\
\text { serum urate* } \\
\text { IRR lung cancer }\end{array}$ & $\mathbf{p ~ v a l u e}$ & Incidence change per 10,000 PYs \\
\hline Overall & $-10.5(-23$ to 2.1$)$ & 0.10 & $0.88(0.68-1.14)$ & 0.33 & $-0.98(-2.95$ to 1.00$)$ \\
Never & $-12.3(-28.5$ to 3.9$)$ & 0.14 & $0.88(0.42-1.85)$ & 0.74 & $-0.25(-1.76$ to 1.26$)$ \\
Former & $-12.0(-32.9$ to 8.9$)$ & 0.26 & $0.86(0.59-1.26)$ & 0.43 & $-1.36(-4.75$ to 2.03$)$ \\
Current & $11.0(-32.9$ to 55.0$)$ & 0.62 & $0.91(0.61-1.34)$ & 0.62 & $-3.40(-17.01$ to 10.21$)$ \\
Overall regular & $-5.0(-28.9$ to 18.8$)$ & 0.68 & $0.88(0.66-1.18)$ & 0.40 & $-2.43(-8.09$ to 3.24$)$ \\
Light former & $-14.3(-50.7$ to 22.2$)$ & 0.44 & $1.60(0.69-3.70)$ & 0.27 & $3.63(-2.83$ to 10.08$)$ \\
Heavy former & $-17.6(-54.0$ to 18.8$)$ & 0.34 & $0.68(0.42-1.10)$ & 0.12 & $-6.11(-13.73$ to 1.52$)$ \\
Current light & $15.9(-43.5$ to 75.3$)$ & 0.60 & $1.00(0.54-1.86)$ & 1.00 & $0.05(-23.32$ to 23.43$)$ \\
Current heavy & $40.9(-37.7$ to 119.4$)$ & 0.31 & $0.56(0.31-1.00)$ & 0.05 & $-39.10(-78.36$ to 0.16$)$ \\
\hline
\end{tabular}

\section{IRR incidence rate ratio}

*Adjusted for sex, age, calendar year, ethnicity (first 40 principal components) and recruitment centre 
example, tissue hypoxia and inflammation can induce urate production by the degradation of adenosine triphosphate. Residual confounding due to omission or mismeasurement of causal variables could also explain the negative association between urate and $\mathrm{FEV}_{1}$. We found L-shaped associations between urate and lung cancer incidence in current and regular smokers with the predicted incidence highest in those with the greatest number of pack-years of smoking. A case-cohort study of urate and cancer reported negative associations with breast and cancer mortality, and weak negative trends for lung cancer that substantially weakened after adjustment [31]. No interactions were found with other variables including smoking status, although the smaller number of cases $(n=195)$ may have reduced precision of these estimates.

A cross-sectional study reported improved $\mathrm{FEV}_{1}$ in post-menopausal women with the SLC2A9 variant (rs11722228) variant associated with raised urate, suggesting a role for female hormones in urate antioxidant activity [32]. In contrast, a recent phenome-wide association study (PheWAS) in UK biobank indicated unspecified diseases of the respiratory system were potentially causally increased in older women with genetically raised urate [33]. A large MR found no support for a causal association between genetically raised urate with lung function, higher risk of respiratory symptoms or COPD [34]. There were no clear interactions with sex and smoking status. A comprehensive review of hundreds of studies of urate including meta-analyses of observational, MR and randomised controlled trials, concluded there was only robust evidence of a positive association with gout and nephrolithiasis [35]. Our results for urate contrast with our recent findings for another endogenous antioxidant bilirubin [15]. In this case we found evidence of a causal relationship with lung cancer that increased in strength with smoking exposure.

\section{Strengths and limitations}

The strengths of the present study are the large sample size, the longitudinal analysis for lung cancer and the availability of data on many potential confounders. The limitations include the use of self-report for smoking status, the short length of follow-up and potential for selection bias. UK Biobank participants are healthier compared to the wider population and the rates of smoking-related diseases, in particular, are substantially lower, which could lead to selection bias [36]. For example, suppose people with genetically low urate are less likely to recruited into UK Biobank due to poor health or death. In that case, the result could be an underestimation of the observational and causal associations. The selected genetic variants explained $5 \%$ of the variance in urate, which may have been too low to calculate precise causal estimates for lung cancer. Even larger cohorts than UK Biobank with a younger age at recruitment are needed to robustly exclude a causal association with lung cancer. However, we also found no supporting evidence of a causal relationship with the continuous outcome $\mathrm{FEV}_{1}$, which we might expect if urate is an important antioxidant. Although we found no strong evidence of a causal association with urate present in blood serum, this does not exclude an antioxidant role for urate found at high levels in the respiratory lining fluid.

\section{Conclusions}

Self-reported current/regular smokers participating in UK Biobank with low levels of serum urate had higher rates of lung cancer and higher $\mathrm{FEV}_{1}$. Our findings using the Mendelian randomization approach, taken together with the existing literature, suggest serum urate is unlikely to represent a modifiable risk factor relevant to adult respiratory health and disease.

\section{Abbreviations \\ $\mathrm{FEV}_{1}$ : Forced expiratory volume per second; IRR: Incidence rate ratio; MR: Mendelian randomization; PY: Person years.}

\section{Supplementary Information}

The online version contains supplementary material available at https://doi. org/10.1186/s12931-021-01768-y.

Additional file 1: Figure S1. Observational associations between serum urate and lung cancer incidence by smoking status and sex after adding one and two years between the urate test date and cohort entry. Figure S2. Observational associations between serum urate and FEV 1 by sex adjusted for age, calendar year, ethnicity (first 40 principal components), height and recruitment centre (Model 1) and additionally for weight (Model 2). Non-linear relationships were captured using restricted cubic spline transformation with three knots placed at the 10th, 50th and 90th percentiles of urate levels. Figure S3. Observational associations between serum urate and $\mathrm{FEV}_{1}$ with interactions with smoking packyears in regular smokers by sex adjusted for age, calendar year, ethnicity (first 40 principal components), height, recruitment centre (Model 1) and additionally for weight (Model 2). Figure S4. Per allele effects on serum urate, baseline $\mathrm{FEV}_{1}$ and lung cancer incidence. Table S1. Association between genetically predicted urate using rs12498742 and rs2231142, FEV 1 and lung cancer incidence. Table S2. The genetically instrumented cross-sectional relationships between urate and other outcomes

\section{Acknowledgements}

The views expressed are those of the authors and not necessarily those of the National Health Service, the National Institute for Health Research or the Department of Health and Social Care.

\section{Authors' contributions}

LJH \& IN contributed to the study design. LJH conducted statistical analyses. LJH wrote the initial draft of the manuscript. All authors participated in the data interpretation and contributed to the final draft of the manuscript with intellectual importance. All authors read and approved the final manuscript. 


\section{Funding}

This research was funded in whole, or in part, by the Wellcome Trust [Grant number 209207/Z/17/Z]. For the purpose of open access, the author has applied a CC BY public copyright licence to any Author Accepted Manuscript version arising from this submission. Ian Hall is a recipient of an NIHR Senior Investigator award.

\section{Availability of data and materials}

The data that support the findings of this study are available from UK Biobank but restrictions apply to the availability of these data, which were used under license for the current study, and so are not publicly available. Data are however available from the authors upon reasonable request and with permission of UK Biobank.

\section{Declarations}

\section{Ethics approval}

UK Biobank received ethics approval from the National Health Service National Research Ethics Service (Ref: 11/NW/0382).

\section{Consent for publication}

Not required.

\section{Competing interests}

The authors declare that they have no competing interests.

\section{Author details}

${ }^{1}$ Research Department of Primary Care and Population Health, University College London, Royal Free Hospital Campus, London NW3 2PF, UK. ${ }^{2}$ University of Nottingham, 6123, Division of Respiratory Medicine, Nottingham, Nottinghamshire, UK. ${ }^{3}$ National Institute for Health Research Nottingham BRC, Nottingham, UK

Received: 4 January 2021 Accepted: 2 June 2021

Published online: 16 June 2021

\section{References}

1. Varela-Echavarria A, Montes de Oca-Luna R, Barrera-Saldana HA. Uricase protein sequences: conserved during vertebrate evolution but absent in humans. FASEB J. 1988;2(15):3092-6.

2. Keilin J. The biological significance of uric acid and guanine excretion. Biol Rev. 1959:34(3):265-94

3. Ames BN, Cathcart R, Schwiers E, Hochstein P. Uric acid provides an antioxidant defense in humans against oxidant- and radical-caused aging and cancer: a hypothesis. Proc Natl Acad Sci USA. 1981;78(11):6858-62.

4. Oda M, Satta Y, Takenaka O, Takahata N. Loss of urate oxidase activity in hominoids and its evolutionary implications. Mol Biol Evol. 2002;19(5):640-53.

5. Wu XW, Muzny DM, Lee CC, Caskey CT. Two independent mutational events in the loss of urate oxidase during hominoid evolution. J Mol Evol. 1992:34(1):78-84

6. Frei B, Stocker R, Ames BN. Antioxidant defenses and lipid peroxidation in human blood plasma. Proc Natl Acad Sci USA. 1988:85(24):9748-52.

7. Glantzounis GK, Tsimoyiannis EC, Kappas AM, Galaris DA. Uric acid and oxidative stress. Curr Pharm Des. 2005:11(32):4145-51.

8. Maxwell SR, Thomason H, Sandler D, Leguen C, Baxter MA, Thorpe GH, et al. Antioxidant status in patients with uncomplicated insulin-dependent and non-insulin-dependent diabetes mellitus. Eur J Clin Invest. 1997;27(6):484-90.

9. Becker BF. Towards the physiological function of uric acid. Free Radical Biol Med. 1993;14(6):615-31
10. Housley DG, Mudway I, Kelly FJ, Eccles R, Richards RJ. Depletion of urate in human nasal lavage following in vitro ozone exposure. Int J Biochem Cell Biol. 1995:27(11):1153-9.

11. van der Vliet A, O'Neill CA, Cross CE, Koostra JM, Volz WG, Halliwell B, et al. Determination of low-molecular-mass antioxidant concentrations in human respiratory tract lining fluids. Am J Physiol. 1999;276(2 Pt 1):L289-96.

12. Horsfall LJ, Nazareth I, Petersen I. Serum uric acid and the risk of respiratory disease: a population-based cohort study. Thorax. 2014;69(11):1021-6.

13. Sudlow C, Gallacher J, Allen N, Beral V, Burton P, Danesh J, et al. UK Biobank: an open access resource for identifying the causes of a wide range of complex diseases of middle and old age. PLoS Med. 2015:12(3):e1001779.

14. Bycroft C, Freeman C, Petkova D, Band G, Elliott LT, Sharp K, et al. Genome-wide genetic data on 500,000 UK Biobank participants. bioRxiv. 2017:166298

15. Horsfall LJ, Burgess S, Hall I, Nazareth I. Genetically raised serum bilirubin levels and lung cancer: a cohort study and Mendelian randomisation using UK Biobank. Thorax. 2020;75(11):955-64.

16. Hanscombe KB, Coleman JRI, Traylor M, Lewis CM. ukbtools: an R package to manage and query UK Biobank data. PLoS ONE. 2019;14(5):e0214311.

17. Kottgen A, Albrecht E, Teumer A, Vitart V, Krumsiek J, Hundertmark C, et al Genome-wide association analyses identify 18 new loci associated with serum urate concentrations. Nat Genet. 2013:45(2):145-54.

18. Hoque KM, Dixon EE, Lewis RM, Allan J, Gamble GD, Phipps-Green AJ et al. The ABCG2 Q141K hyperuricemia and gout associated variant illuminates the physiology of human urate excretion. Nat Commun. 2020;11(1):2767.

19. Brown KF, Rumgay H, Dunlop C, Ryan M, Quartly F, Cox A, et al. The fraction of cancer attributable to modifiable risk factors in England, Wales, Scotland, Northern Ireland, and the United Kingdom in 2015. Br J Cancer. 2018;118(8):1130-41.

20. Wain LV, Shrine N, Miller S, Jackson VE, Ntalla I, Soler Artigas M, et al. Novel insights into the genetics of smoking behaviour, lung function, and chronic obstructive pulmonary disease (UK BiLEVE): a genetic association study in UK Biobank. Lancet Respir Med. 2015;3(10):769-81.

21. Burgess S, Thompson SG, Burgess S. Mendelian randomization: methods for using genetic variants in causal estimation. Boca Raton, FL: CRC Press, Taylor \& Francis Group; 2015. 1 online resource: illustrations. P.

22. Burgess S, Davey Smith G, Davies N, Dudbridge F, Gill D, Glymour M et al. Guidelines for performing Mendelian randomization investigations [version 1; peer review: awaiting peer review]. Wellcome Open Res. 2019:4:186

23. Wade KH, Carslake D, Sattar N, Davey Smith G, Timpson NJ. BMI and mortality in UK Biobank: revised estimates using Mendelian randomization. Obesity (Silver Spring). 2018;26(11):1796-806.

24. Narang RK, Topless R, Cadzow M, Gamble G, Stamp LK, Merriman TR, et al. Interactions between serum urate-associated genetic variants and sex on gout risk: analysis of the UK Biobank. Arthritis Res Ther. 2019;21(1):13.

25. Weber S. bacon: an effective way to detect outliers in multivariate data using Stata (and Mata). Stata J. 2010;10(3):331-8.

26. Royston P. marginscontplot: plotting the marginal effects of continuous predictors. Stata J. 2013;13(3):510-27.

27. Raymakers AJN, Mayo J, Lam S, FitzGerald JM, Whitehurst DGT, Lynd LD. Cost-effectiveness analyses of lung cancer screening strategies using low-dose computed tomography: a systematic review. Appl Health Econ Health Policy. 2016;14(4):409-18.

28. Kahnert K, Alter P, Welte T, Huber RM, Behr J, Biertz F, et al. Uric acid, lung function, physical capacity and exacerbation frequency in patients with COPD: a multi-dimensional approach. Respir Res. 2018;19(1):110.

29. Aida Y, Shibata Y, Osaka D, Abe S, Inoue S, Fukuzaki K, et al. The relationship between serum uric acid and spirometric values in participants in a health check: the Takahata study. Int J Med Sci. 2011:8(6):470-8. 
30. Bartziokas K, Papaioannou Al, Loukides S, Papadopoulos A, Haniotou A, Papiris $S$, et al. Serum uric acid as a predictor of mortality and future exacerbations of COPD. Eur Respir J. 2014;43(1):43-53.

31. Kuhn T, Sookthai D, Graf ME, Schubel R, Freisling H, Johnson T, et al. Albumin, bilirubin, uric acid and cancer risk: results from a prospective population-based study. Br J Cancer. 2017;117(10):1572-9.

32. Fujikawa H, Sakamoto Y, Masuda N, Oniki K, Kamei S, Nohara H, et al. Higher blood uric acid in female humans and mice as a protective factor against pathophysiological decline of lung function. Antioxidants (Basel). 2020;9(5):387

33. Li X, Meng X, Spiliopoulou A, Timofeeva M, Wei WQ, Gifford A, et al. MR-PheWAS: exploring the causal effect of SUA level on multiple disease outcomes by using genetic instruments in UK Biobank. Ann Rheum Dis. 2018;77(7):1039-47.

34. Kobylecki CJ, Vedel-Krogh S, Afzal S, Nielsen SF, Nordestgaard BG. Plasma urate, lung function and chronic obstructive pulmonary disease: a
Mendelian randomisation study in 114979 individuals from the general population. Thorax. 2018;73(8):748-57.

35. Li X, Meng X, Timofeeva M, Tzoulaki I, Tsilidis KK, loannidis JP, et al. Serum uric acid levels and multiple health outcomes: umbrella review of evidence from observational studies, randomised controlled trials, and Mendelian randomisation studies. Bmj. 2017;357:j2376.

36. Fry A, Littlejohns TJ, Sudlow C, Doherty N, Adamska L, Sprosen T, et al. Comparison of sociodemographic and health-related characteristics of UK Biobank participants with those of the general population. Am J Epidemiol. 2017;186(9):1026-34

\section{Publisher's Note}

Springer Nature remains neutral with regard to jurisdictional claims in published maps and institutional affiliations.
Ready to submit your research? Choose BMC and benefit from:

- fast, convenient online submission

- thorough peer review by experienced researchers in your field

- rapid publication on acceptance

- support for research data, including large and complex data types

- gold Open Access which fosters wider collaboration and increased citations

- maximum visibility for your research: over $100 \mathrm{M}$ website views per year

At BMC, research is always in progress.

Learn more biomedcentral.com/submissions 